Document downloaded from:

http://hdl.handle.net/10251/38952

This paper must be cited as:

Climent, S.; Sánchez Matías, AM.; Blanc Clavero, S.; Capella Hernández, JV.; Ors Carot, R. (2013). Wireless sensor networks with energy harvesting: Modeling and simulation based on a practical architecture using real radiation levels. Concurrency and Computation: Practice and Experience. 1-19. doi:10.1002/cpe.3151.

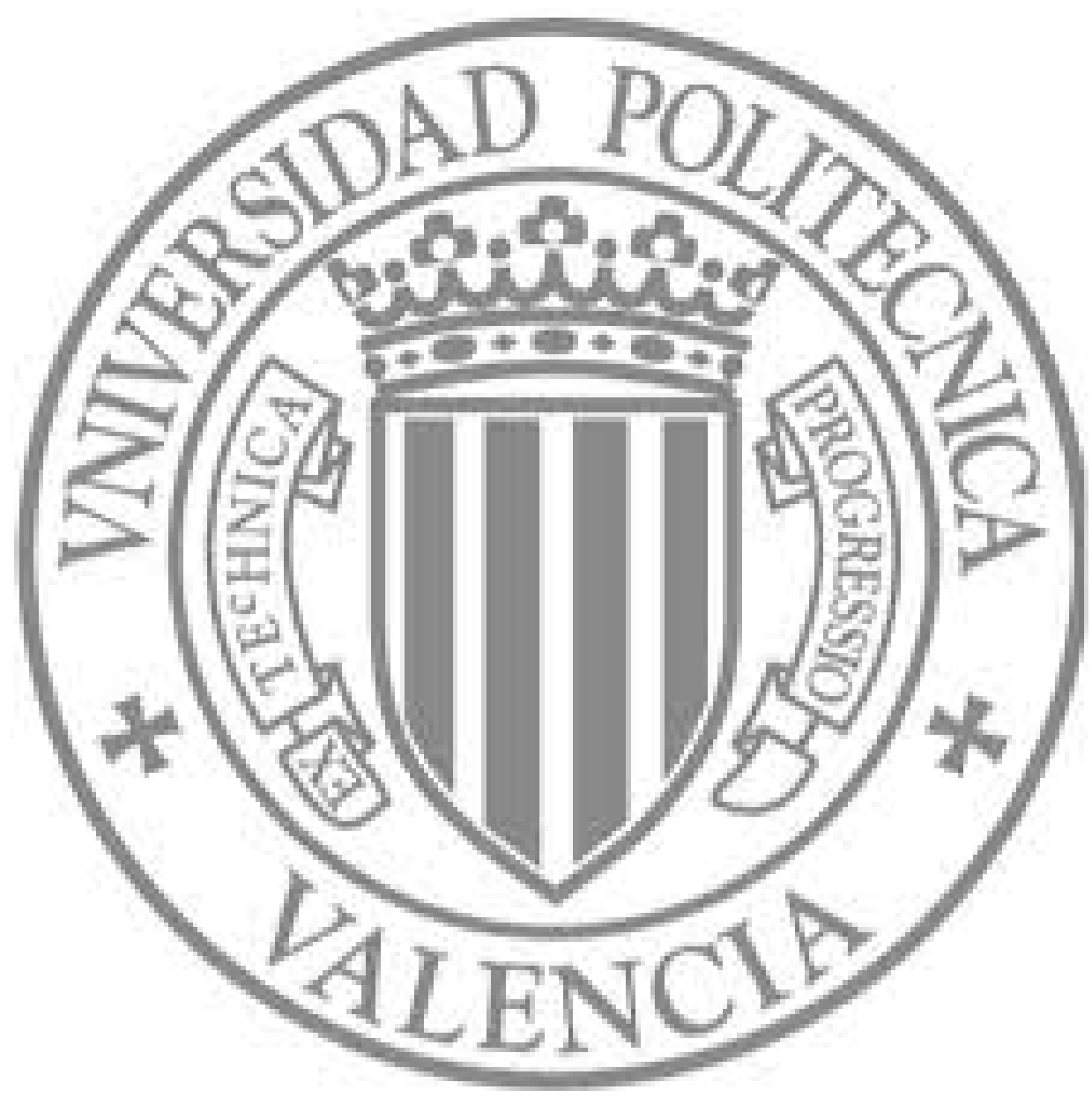

The final publication is available at

http://onlinelibrary.wiley.com/doi/10.1002/cpe.3151/pdf

Copyright Wiley 


\title{
Wireless sensor network with energy harvesting: modeling and simulation based on a practical architecture using real radiation levels
}

\author{
S. Climent, A. Sanchez, S. Blanc, J. V. Capella and R. Ors*,† \\ Universitat Politècnica de València, Insitut ITACA. Camí. de Vera s/n. 46022 Valencia, Spain
}

\begin{abstract}
SUMMARY
This paper presents a new energy-harvesting model for a network simulator that implements super-capacitor energy storage with solar energy-harvesting recharge. The model is easily extensible, and other energyharvesting systems, or different energy storages, can be further developed. Moreover, code can be conveniently reused as the implementation is entirely uncoupled from the radio and node models. Real radiation data are obtained from available online databases in order to dynamically calculate super-capacitor charge and discharge. Such novelty enables the evaluation of energy evolution on a network of sensor nodes at various physical world locations and during different seasons. The model is validated against a real and fully working prototype, and good result correlation is shown. Furthermore, various experiments using the ns-3 simulator were conducted, demonstrating the utility of the model in assisting the research and development of the deployment of everlasting wireless sensor networks.
\end{abstract}

KEY WORDS: $\quad$ wireless sensor networks; energy harvesting; ns-3; energy models; simulation

\section{INTRODUCTION}

A wireless sensor network (WSN) consists of a number of units, called sensor nodes, that use a wireless medium to communicate [1]. Sensor nodes are small and usually self-powered. Much research on WSNs has been focused on prolonging the lifetime of these nodes with an energy-efficient use of their batteries.

It is currently viable to prolong the lifetime of a sensor node indefinitely using energy-harvesting techniques combined with low-power architectures and energy-aware protocols. Energy-harvesting techniques are used to collect energy from ambient sources and produce electrical energy that is stored in a buffer. These techniques are growing in importance because of their falling prices and improved technical performance - as well as accumulated scientific experience [2,3].

In this paper, we present and validate an energy-harvesting model based on solar power and super capacitors for the ns-3 network simulator. The model can be easily extended to include new mechanical $[4,5]$ or thermal $[6,7]$ energy sources, as well as different storage modules such as batteries (although the ns-3 already includes a Li-ion battery model). Moreover, because the implementation is completely uncoupled from the radio model, it can be easily included with the already available models for network communication - and this enables us to simulate network behavior by only modifying the simulation scripts.

\footnotetext{
*Correspondence to: R. Ors, Universitat Politècnica de València, Insitut ITACA. Camí. de Vera s/n. Edifici 8G. 46022 Valencia, Spain

†E-mail: rors@disca.upv.es
} 
The result is a dynamic energy model because the energy available in the buffer is continuously recalculated to reflect solar radiation and the current node operating mode. The energy model uses an event-driven schedule to update radio mode changes notified by the radio model.

Moreover, it is possible to simulate several days, weeks, months, or even years, using real solar radiation curves observed in specific world locations. These data can be easily obtained from meteorological databases. For example, the authors have used radiation levels observed by AEMet [8] in Spain or by the PANGAEA [9] project in Alaska (USA) and Tõravere (Estonia).

The presented research has an important utility in the future study and development of everlasting WSNs. This paper proposes the evaluation of reception and transmission rates or sleep periods with respect to the impact of their configuration at different locations.

This paper is organized as follows: In Section 2, the related work is introduced. In Section 3, the simulation model is introduced and explained in detail. An exhaustive validation is performed in Section 4. In Section 5, the utility of the model is demonstrated by showing how it can be used when dimensioning the actual hardware node before its deployment. Moreover, in Section 6, a simple energy-neutral policy is evaluated in two world regions over 1 year. Finally, conclusions and future work are presented in Section 7.

\section{RELATED WORK}

Current state of the art in energy-harvesting WSNs is focused on modeling and designing energyharvesting circuits $[10,11]$. However, there has been little work performed on the integration of these models into a WSN simulator. Because network simulators are not well prepared for simulating nodes with energy-harvesting capabilities, the various networking protocols that have been proposed usually perform some type of simplification - such as assuming a uniformly distributed random variable [12] or even constant replenishment [13,14]. In [15], the authors model the energy flow at different rates or according to different on-off processes. However, it can be difficult to describe the energy variations over long periods of time at different world locations.

Various frameworks are used to carry out power-profiling estimation of hardware [16]. However, it can be difficult to model a whole network with hundreds of nodes and numerous communication protocols (collisions, backoff time, etc.).

Power aware wireless sensor [17] is a simulation framework developed on top of the OMNeT++ network simulator. Only basic, but energy-aware, simulator models are available. WSNSim [18] is another energy-aware network simulator built from scratch. WSNSim includes various energyrelated models such as the following: a solar panel energy model, a battery model, and a super-capacitor model. However, accuracy has not been demonstrated.

Another simulation tool has been proposed by De Mil et al. and developed over the Castalia network simulator [19]. It provides solar panel and battery models and validates them with prototype nodes. However, the prototype nodes use software to emulate the solar panel and super capacitor.

Finally, another framework is presented in [20]. However, modeling protocols other than time division multiple access can be complex because the model needs to know how much time a node will be in the various radio states.

\section{NS-3 ENERGY MODEL}

This section introduces the implementation of an energy-harvesting model for the ns-3 simulator. The model architecture is based on a typical energy-harvesting circuit - [11,21-24] - such as that depicted in Figure 1. Further details on how the different formulae have been derived can be obtained in [25]. Figure 2 depicts a simplified class diagram of the involved classes, and Figure 3 shows the corresponding block diagram. The block diagram illustrates how the solar panel block is connected to the CapacitorEnergySource module and how this, in turn, is connected to a RadioEnergyModel and a SensorEnergyModel.

The SolarPanelEnergyModel class provides energy (when it is able to harvest it) to the CapacitorEnergySource. This module calculates and manages the available energy on the actual capacitor by taking into account its voltage, the current provided by the solar panel, and the current consumed 


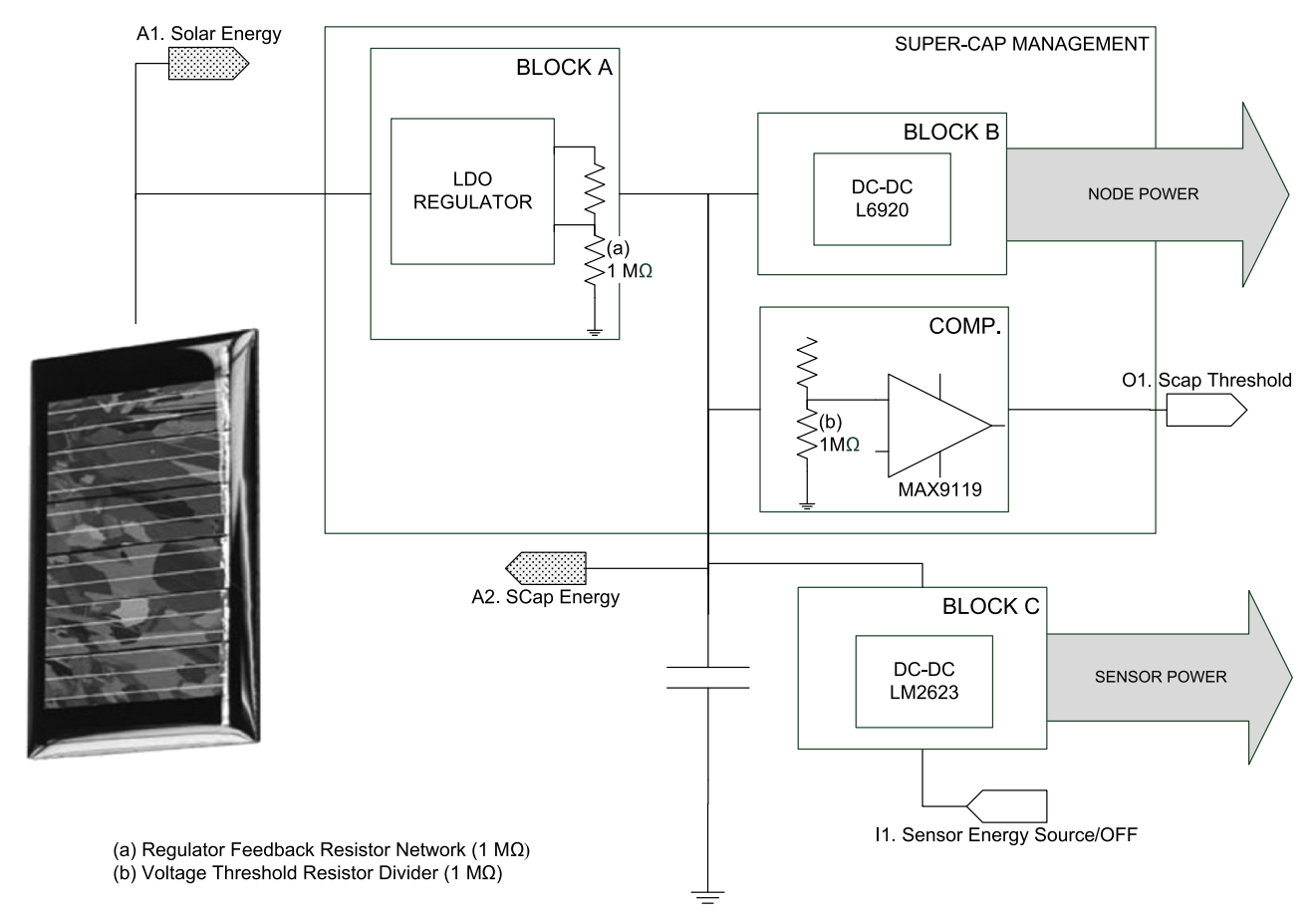

Figure 1. Energy-harvesting module diagram block.

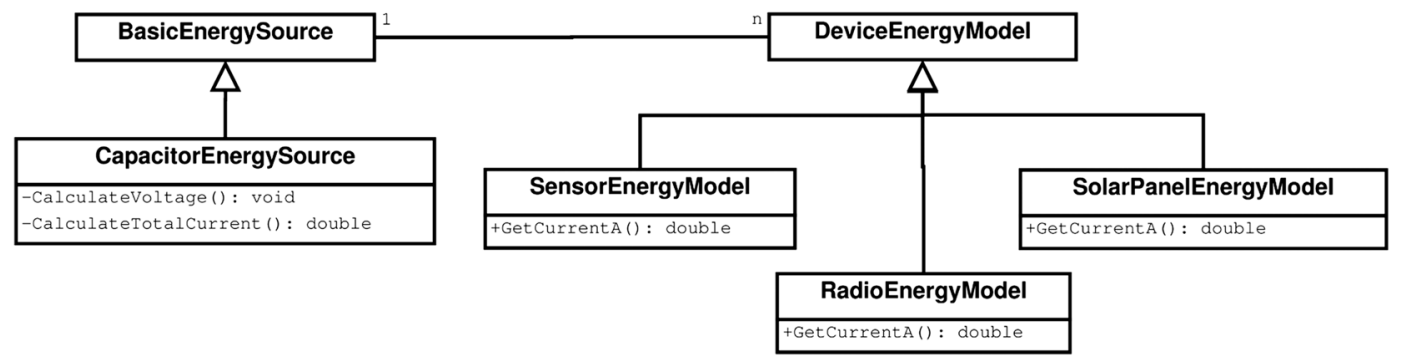

Figure 2. Simplified class diagram of the ns- 3 energy model.

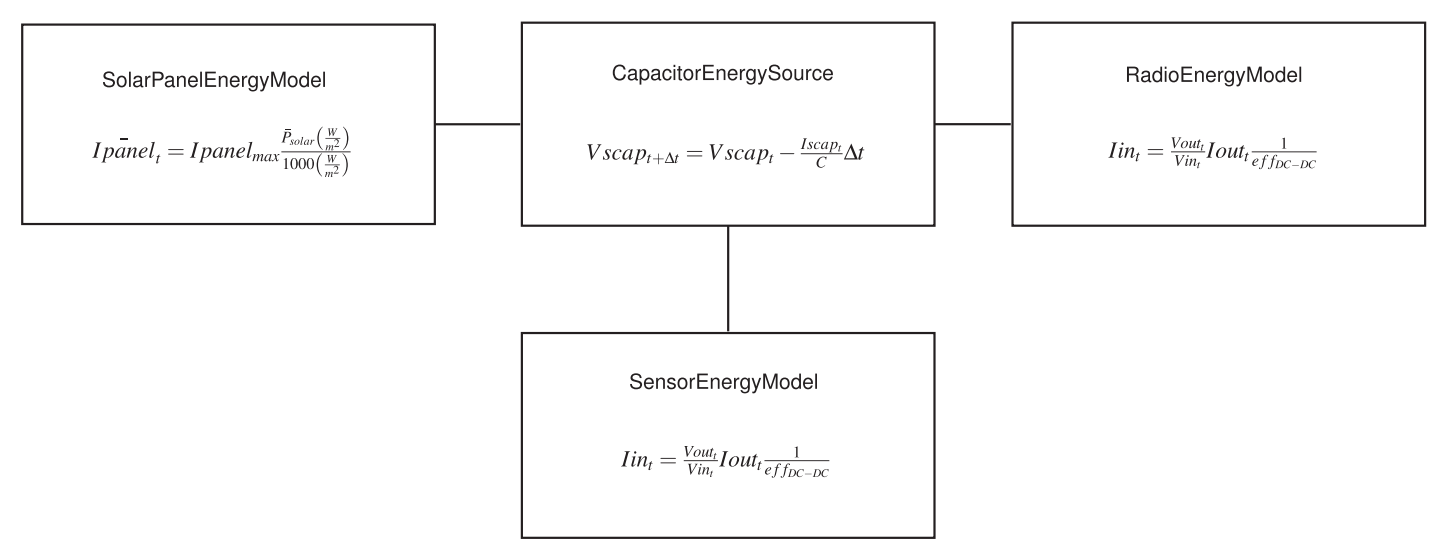

Figure 3. Energy-harvesting module diagram block. 
by the different elements of the node. In this case, we have developed radio and sensor energy models; however, other models can be developed and easily integrated.

\subsection{Capacitor energy source}

Previous energy-based approaches calculate available capacitor energy by adding and subtracting harvested and consumed energy, respectively [18, 26, 27]. However, as Renner et al. [28] demonstrate, this energy depends on instant voltage and current conditions. Hence, this model accurately calculates the remaining super-capacitor energy using current and voltage values. Expression (1) calculates the super-capacitor voltage variations, where $C$ is the actual capacitor capacity, Vscap is the capacitor voltage at time $t$, and $\Delta t$ is the minimum time increment.

$$
\operatorname{Vscap}_{t+\Delta t}=\operatorname{Vscap}_{t}-\int_{t}^{t+\Delta t} \frac{\operatorname{Iscap}_{t}}{C} \partial t
$$

Iscap is the net current that arrives at the super capacitor. It depends on different circuit parameters, but it can be simply calculated as the difference between the current demanded by the super capacitor (Idis) and the current coming from the energy source (Ipanel), (2).

$$
\text { Iscap }_{t}=\text { Idis }_{t}-\text { Ipanel }_{t}
$$

Assuming that time intervals $(\delta t)$ are short enough to guarantee nearly constant current (Iscap) during the interval, equation (3) is used to describe the super-capacitor voltage level instead of the differential equation (1).

$$
\operatorname{Vscap}_{t+\Delta t}=\operatorname{Vscap}_{t}-\frac{\text { Iscap }_{t}}{C} \Delta t
$$

Expression (3) is calculated iteratively to progressively obtain the super-capacitor voltage from the previous voltage and current consumption. Under this assumption, equation (3) can be seen as an approximation of equation (1) using the midpoint integration method. The error of applying the midpoint numerical integration method is bounded by expression (4).

$$
E_{V s c a p} \leqslant \frac{\Delta t^{3}}{24} \frac{\operatorname{Iscap}^{\prime \prime}(t)}{C}
$$

It can be seen that the shorter the time interval taken for each interval and the larger the capacitance chosen, the greater the accuracy obtained. Moreover, error decreases if the super-capacitor current is constant.

Taking a look at the actual model implementation, the CalculateVoltage method returns the voltage, calculated using expression (3), that a capacitor can deliver after $\Delta t$. This $\Delta t$ is a trade-off between simulation accuracy and efficiency and must be configured. In addition, the capacitor voltage is always updated whenever a change in the radio and sensor model occurs (or any other connected model).

Component demands and current delivered by the solar panels are calculated using the CalculateTotalCurrent method, which, at the same time, calls to the GetCurrentA method for the current that is being used (or delivered in the case of the solar model) by each component. Both demanded and delivered currents are added using expressions (2) to update capacitor voltage.

As stated earlier, the CalculateVoltage method is always called when a mode change occurs at either radio or sensor models. Any change causes an event to recalculate capacitor energy based on the previous state (mode) of the radio (or sensor) and the amount of time since the previous calculation was performed. However, if no event is triggered during a period $\Delta t$, an automatic call to CalculateVoltage is made in order to maintain simulation accuracy.

\subsection{Radio energy model}

This class models the energy consumption of a radio transceiver. Basically, it calculates the actual current consumption of the radio transceiver and communicates with the super-capacitor model. 
To uncouple the implementation of the radio transceiver from the implementation of the energy model, the radio transceiver makes callbacks about its state transitions to the energy model. Hence, this radio energy model can be reused with different models of a radio transceiver by simply adjusting the consumption parameters and available states.

The current implementation includes four different states (RX, TX, IDLE, and SLEEP), and a configurable amount of current can be set for each state (Table I). Moreover, because the capacitor voltage varies over time depending on its actual charge and a node needs a fairly constant voltage to correctly operate, a Direct Current DC-DC converter is included, and its efficiency is taken into account when calculating the final current consumption.

Expression (5) is used to calculate the actual current consumption of the radio transceiver by taking into account the current consumed by the radio transceiver and the efficiency of the DC-DC converter.

$$
\operatorname{Iin}_{t}=\frac{\text { Vout }_{t}}{\operatorname{Vin}_{t}} \text { Iout }_{t} \frac{1}{\text { eff }_{D C-D C}}
$$

where Vout corresponds to the node operational voltage, Vin to the current capacitor voltage, Iout to the current consumed by the radio transceiver (which depends on the operational mode), and $e f f_{D C-D C}$ to the efficiency of the DC-DC converter.

\subsection{Sensor energy model}

This class models the energy consumption of a sensor attached to the node. This model is fundamentally the same as the radio energy model, but with only two operational modes: enabled and disabled.

In the enabled state, the sensor consumes a configurable amount of current, while consuming zero in the disabled state. Final current consumption is estimated taking into account the consumption of the DC-DC converter by using expression (5).

Hence, expression (5) is reused to calculate current sensor consumption by setting Iout to the current consumed by the sensor.

\subsection{Solar panel energy model}

This class models the current delivered by solar panels. This model receives as a parameter a file containing the integrated global radiation values $\left(\frac{\mathrm{J}}{\mathrm{m}^{2}}\right)$ per each simulated hour.

Hourly-integrated global radiation values can be easily obtained from solar radiation databases and directly imported into the simulation tool. This procedure obtains more realistic scenarios for location conditions and meteorological variability during the day. Clouds or even eclipses can be simulated from real data. Besides, these databases are continuously updated, and historical curves are publicly available.

For example, AEMet weather service database [8] stores hourly-integrated global radiation $\left(\frac{\mathrm{J}}{\mathrm{m}^{2}}\right)$ for several Spanish locations. Average power can be calculated using expression (6), which is a simple ratio between hourly-integrated energy and average power.

$$
\bar{P}_{\text {solar }}\left(\frac{W}{m^{2}}\right)=\frac{\bar{E}_{\text {solar (hourly) }\left(\frac{J}{m^{2}}\right)}}{3600(s)}
$$

Another example is the PANGAEA database [9], which stores hourly-averaged direct and indirect radiation power. Assuming no reflections, hourly-averaged global radiation is the sum of both values.

Finally, actual current delivered by the solar panel is calculated in expression (7). $1000\left(\frac{\mathrm{W}}{\mathrm{m}^{2}}\right)$ corresponds to the solar power density received on the Earth's surface with no clouds at midday and the sun in its zenith ( $\pm 1.5 \%$ error). The model assumes expression (7) using the maximum Ipanel given by the manufacturer [25]. 


$$
\text { Ipanel }_{t}=\text { Ipanel }_{\max } \frac{\bar{P}_{\text {solar }}\left(\frac{W}{m^{2}}\right)}{1000\left(\frac{W}{m^{2}}\right)}
$$

\section{MODEL VERIFICATION}

Verification has been carried out by comparing simulation outcomes with experimental results obtained using a prototyped hardware test bed. To avoid distortions from uncontrollable factors, tests were conducted over deterministic circumstances.

The prototyped hardware nodes have a micro-controller-based design. They consist of a Texas Instruments CC1110 system on chip with its radio transceiver working on the $868 \mathrm{MHz}$ frequency band [29]. Consumption parameters are shown in Table I. Nodes also include a high-precision, low-power ambient temperature sensor catalogued as TI TMP 112 [29] with $6 \mathrm{~mA}$ consumption as shown in Table II.

Hardware nodes also include a 50-farad capacitor with a maximum voltage of $2.3 \mathrm{~V}$. Node operation can start only if the capacitor voltage is above $1 \mathrm{~V}$ and the DC-DC converter efficiency is measured at $92 \%$. In addition, the solar panel included in the prototype delivers $40 \mathrm{~mA}$ maximum current.

These nodes were developed in collaboration with WSNVAL company [30], and an asynchronous wake-up circuit [31] and a sensor interface were also implemented.

\subsection{Solar panel characterization}

The proposed solar panel model has been verified. Figure 4 shows the curves registered by the real prototype and the ns-3 simulator based on AEmet data.

Table I. Radio current consumption.

\begin{tabular}{lc}
\hline Mode & Current \\
\hline TX mode & $30 \mathrm{~mA}$ \\
RX mode & $27 \mathrm{~mA}$ \\
IDLE & $27 \mathrm{~mA}$ \\
SLEEP mode & $12 \mu \mathrm{A}$ \\
\hline
\end{tabular}

Table II. Sensor current consumption.

\begin{tabular}{lc}
\hline Mode & Current $(\mathrm{mA})$ \\
\hline Sensor enabled & 6 \\
Sensor disabled & 0 \\
\hline
\end{tabular}

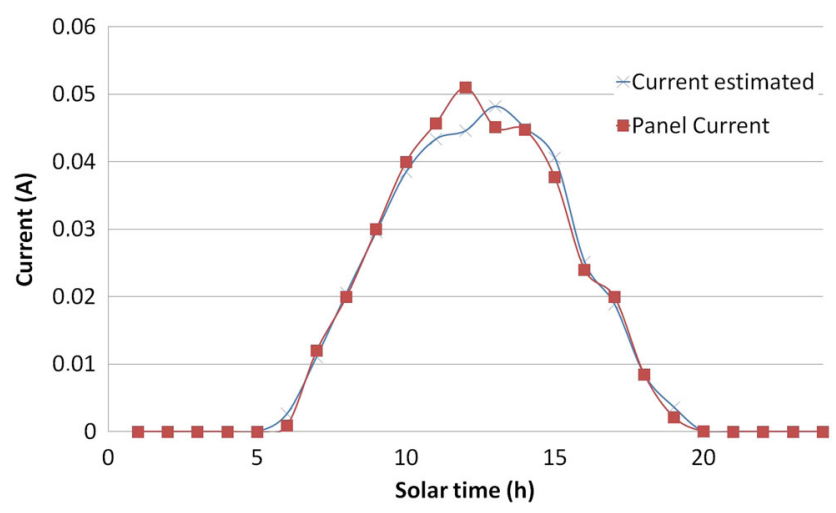

Figure 4. Solar panel current (Ipanel mean per hour). 


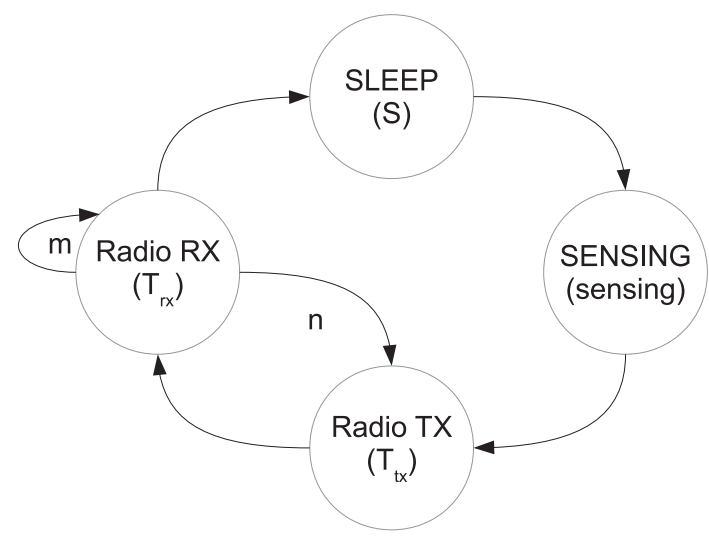

Figure 5. Sensor node simplified state diagram, where each state duration is defined in Table III and S is the node sleep time ( $60 \mathrm{~s}$ by default).

Table III. Three-day test benchmark.

\begin{tabular}{lll}
\hline Task & Symbol & Time \\
\hline Sensing & sensing & $44 \mathrm{~ms}$ \\
Node transmission mode & $t_{T X}$ & $570 \mu \mathrm{s}$ \\
Node receive mode & $t_{R X}$ & $100 \mathrm{~ms}$ \\
\hline
\end{tabular}

The experiment was carried out on May 12, 2011. The prototype was located at the ITACA Institute in Valencia Global Positioning System (GPS) (GPS: 39.478654,-0.333595). Solar radiation data were obtained from the Manises (Valencia) Airport station (GPS: 39.492736,-0.475042).

Although a high correlation is shown, some inaccuracies are observed because of the $10 \mathrm{~km}$ distance between the meteorological station and the ITACA institute. As a consequence, some effects may be shifted in time.

\subsection{Stressful test bench}

Several experiments have been performed discharging the capacitor under different radio energetic demands to verify the model outcomes with the real hardware prototype. The duty cycle is described in Figure 5, where $n$ is the number of TX/RX cycles performed by the radio before returning to SLEEP mode again and $m$ is the number of RX cycles. The sensor is only enabled during SENSING STATE, and during this state, the radio remains in sleep mode.

Three different duty cycles have been tested with 1,2, and 5 sleep time minutes. Receive mode time was established at $500 \mathrm{~ms}$, while a transmission needs $570 \mu \mathrm{s}$. The $n$ and $m$ parameters were set to 0 , and the remaining corresponding values are shown in Table III.

Capacitor energy was continuously powering the system up to 23,35 , and $51 \mathrm{~h}$ and corresponding with 1,2, and 5 min sleep time, respectively. In all the experiments, the capacitor starts with maximum charge.

Figure 6 shows super-capacitor voltage evolution. It can be seen that the proposed analytical model fits the measurements as the maximum error is $2.3 \%$.

\subsection{Three-day outdoor test}

To combine charge and discharge cycles in a real scenario, a three-day benchmark test was carried out. Experiments were run from 2 PM, April 1, 2011, to 10 AM, April 4, 2011. Solar energy conditions are shown in Figure 7.

The node iterates the different states specified in Figure 5 cyclically - with their duration given in Table III. Energy consumption is specified in Tables I and II. 


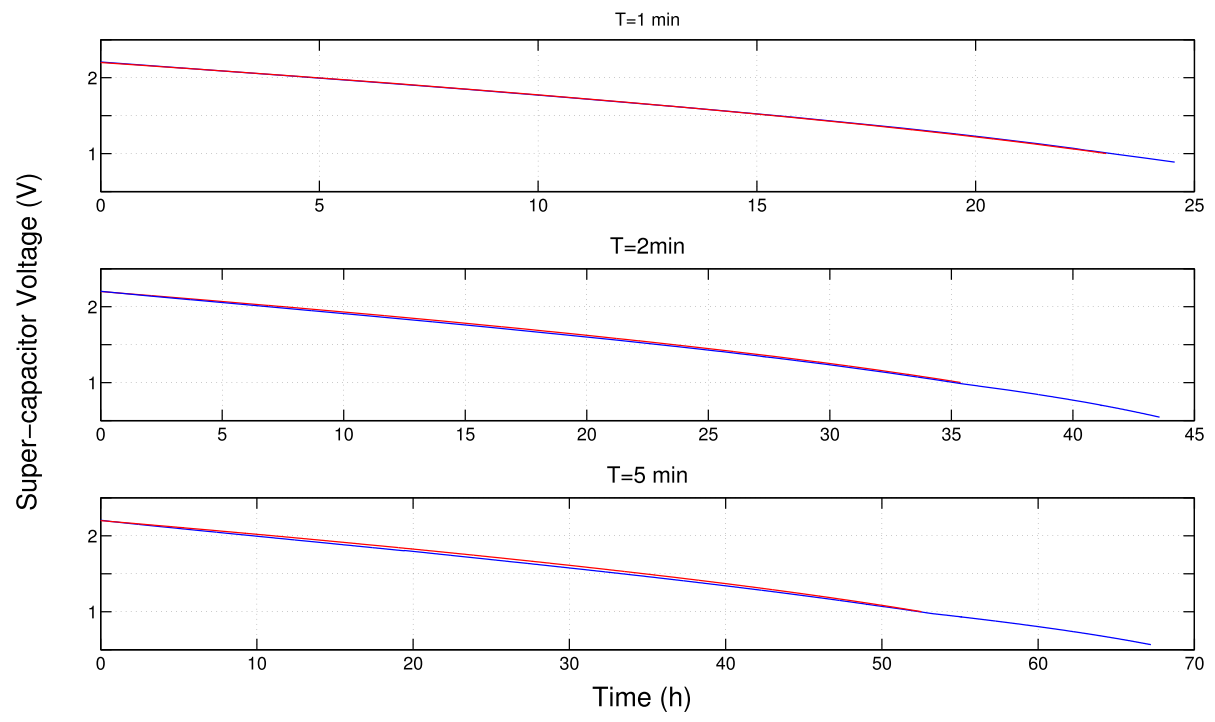

Figure 6. Capacitor voltage in discharge tests: real prototype measures (blue) and ns-3 model (red).

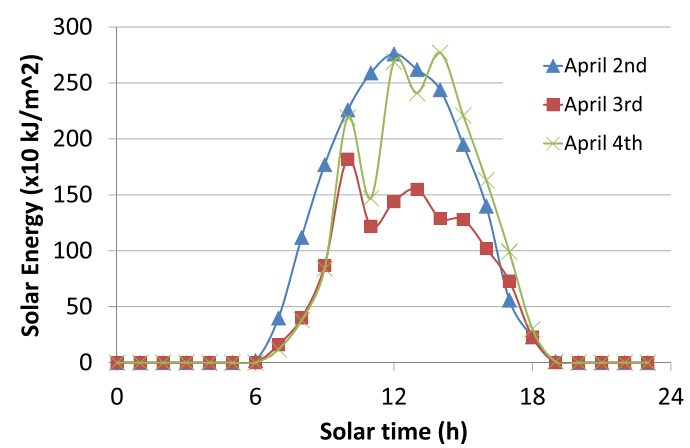

Figure 7. Solar energy conditions during the model validation experiments.

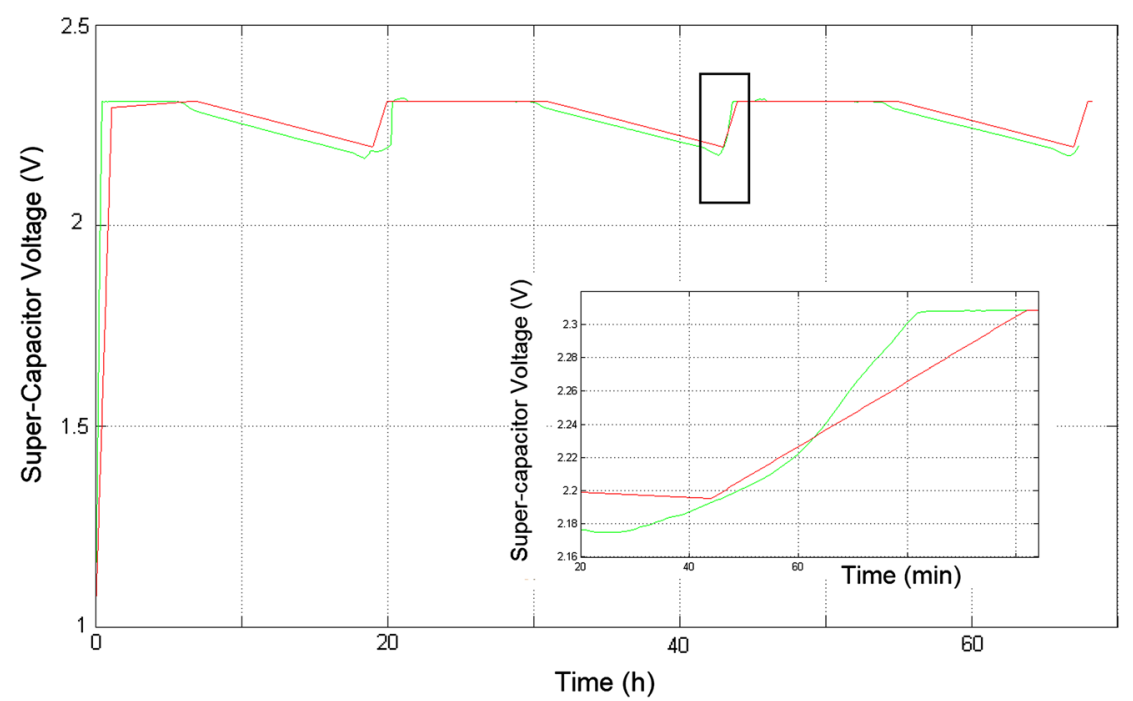

Figure 8. Real test results and model estimation. 
Figure 8 shows a comparison between the real test results and the ns-3 model estimation. Although some inaccuracies are found, both charge and discharge slopes are very similar. However, charge and discharge cycles seem to be shifted in time. This effect is due to the solar database import process. Because solar data offered by AEMet are the energy integrated during a whole hour, the model uses a constant panel supplied current per hour. Consequently, the sun rises earlier in the simulations, and sunset is also delayed.

\section{WIRELESS SENSOR NETWORK SCALING}

Before simulating complex energy-aware protocols, there is a need to predict the maximum current consumption that can be demanded by a network node. The objective is to define energy-neutral strategies during energy-available periods and energy-saving strategies during periods when no energy is available. As an example, several scenarios are proposed in this section to compare energy consumptions. The aim is to show how the proposed model can assist in the discovery of energy depletion conditions and possible unrecoverable energy node failures - and so improve communications reliability.

To perform this evaluation, we have set a basic scenario with the following statements:

(i) Network star topology. Sensor nodes communicate to a single node that acts as network coordinator and packet sink.

(ii) We assume no failures in the network coordinator power supply. The rest of the sensor nodes are powered using the energy-harvesting system presented in this paper.

(iii) ALOHA medium access control protocol. Although throughput can be severely restricted, channel access time is deterministic. No Acknowledgement (ACK) messages are sent in order to minimize network traffic.

(iv) Sensor measurement frequency is configurable in each node.

(v) Nodes can transmit and receive information from the coordinator. For that purpose, sensor nodes will listen to the channel in the duty cycle waiting for incoming packets.

The duty cycle is the same as the one described in Section 4.2 and depicted in Figure 5. If not stated differently, the simulation parameters for these experiments are the following: time for one packet transmission, $0.57 \mathrm{~ms}$; time that a node remains in the idle/RX state, $102.5 \mathrm{~ms}$; time that a node remains in the sleep state, $60 \mathrm{~s}$; and time for sensing, $44 \mathrm{~ms}$. Capacitor capacitance was set to 50 farads, its starting voltage to $0 \mathrm{~V}$, and its maximum voltage to $2.3 \mathrm{~V}$. The node can start its operation only if the capacitor voltage is above $1 \mathrm{~V}$. The efficiency of the DC-DC converter was set to $92 \%$. Solar panel maximum current was set to $40 \mathrm{~mA}$, and the simulation start time was set to match 7 AM solar time. Solar radiation data correspond to radiation measured by AEMet at Valencia (Spain) during April 3, 2011. Simulation stop time was set to $48 \mathrm{~h}$. The sensor is only enabled during sensor-enabled state, and during this state, the radio remains in sleep mode.

With the aim of understanding how the duty cycle affects the node energy consumption and studying the different energy-depletion scenarios (or unrecoverable energy failures), Figure 9 depicts the super-capacitor charge and discharge curves during $48 \mathrm{~h}$ for different TX/RX cycles ( $n$ parameter in Figure 5).

At sunrise, charge curves match similarly in the three cases $(n=10,15$, and 20 cycles). Discharge curves start at sunset and differ for each studied case. A detailed view is shown in Figure 9. With 10 and 15 cycles, the node never stops its operation because capacitor voltage never falls below $1 \mathrm{~V}$, which is the minimum required voltage for the node to maintain operation. At 20 cycles, the energy consumed at night is more than the super capacitor can provide without recharging - and the node stops operation before sunrise.

At sunrise on the second day, the super capacitor starts receiving energy from the solar panel between 7 and $8 \mathrm{AM}$. When performing at 10 cycles, the super capacitor is able to recharge while the node is in operation. However, it can be seen how the discharge continues at 15 cycles, although with a much less pronounced slope. A special case is shown in the 20-cycle simulation. In this case, the node is unable to acquire enough energy to start its operation until 8 AM. 


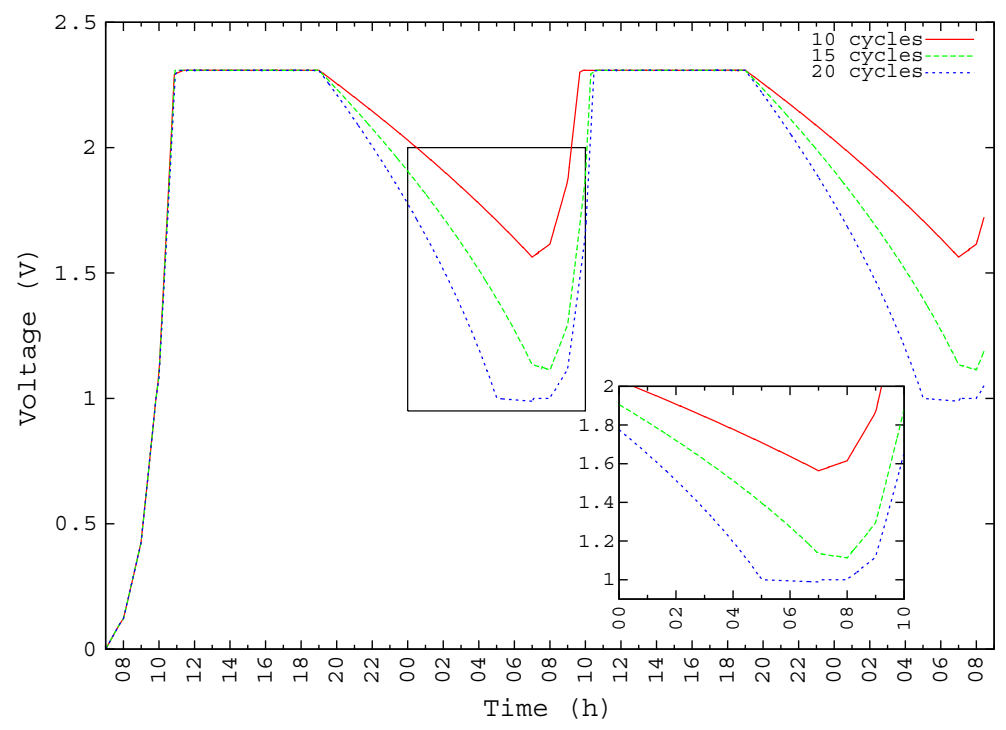

Figure 9. TX/RX cycle variation.

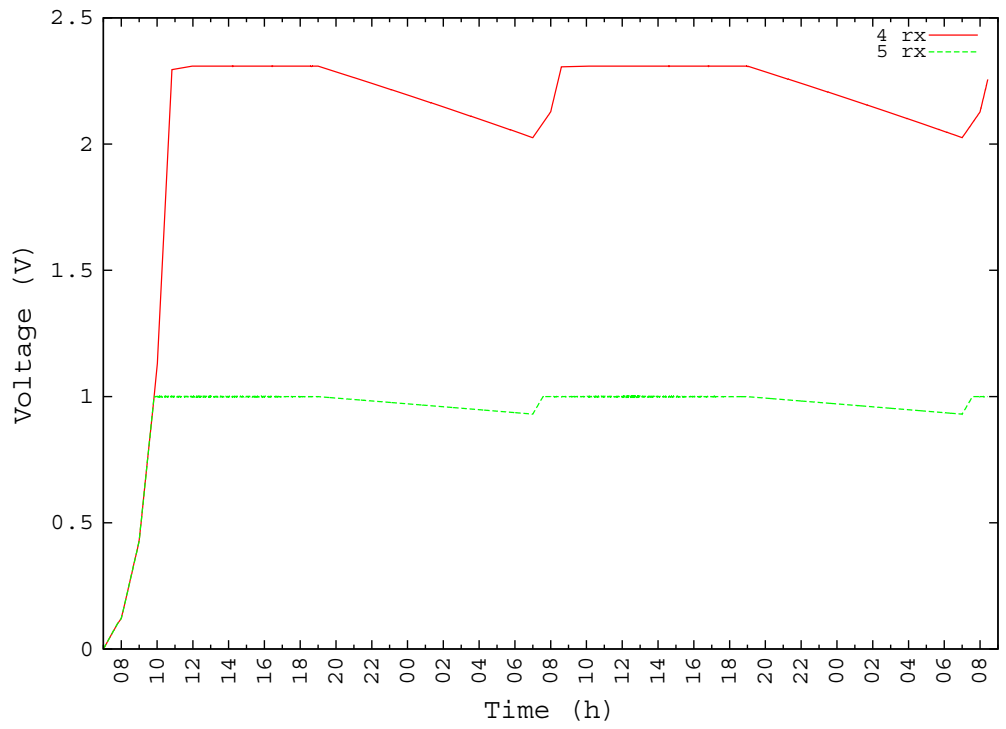

Figure 10. Increasing the RX time by $m=4$ and $m=5$ times without hysteresis.

This effect occurs because the energy demands during this hour are greater than the energy provided by the panel; although the super capacitor is able to recharge, the energy is quickly consumed, and consequently, the node is unable to recover operation until $8 \mathrm{AM}$ - when the solar panel can provide enough energy.

To study this effect, different benchmarks were performed and shown in Figure 10. In this experiment, the duty cycle is modified differently. Instead of varying the $n$ parameter, which effectively modifies the number of TX/RX cycles performed by the node, the $m$ parameter is altered. Hence, while maintaining one TX/RX cycle, the RX state will increase its duration by a factor of $m$. Two different values of $m$ were chosen to illustrate this experiment: 4 and 5 .

When $m=4$, the node is able to perform as normal. However, when the simulation is configured to $m=5$, the solar panel is never able to provide the node with enough energy to start operation. When the capacitor voltage reaches $1 \mathrm{~V}$, the node starts an operative cycle, but the 


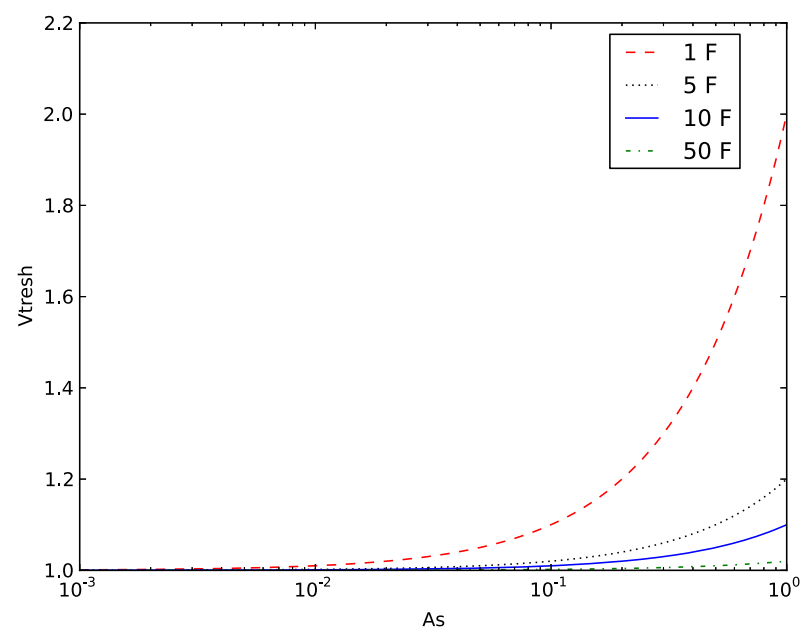

Figure 11. Threshold voltage depending on capacitor capacitance and energy consumption.

radio RX mode consumes too much energy and decreases the capacitor voltage below $1 \mathrm{~V}$ again, producing an unrecoverable energy node failure.

This problem can be easily solved. The key issue is to store enough energy to carry out a complete cycle before the node becomes active, which can be achieved by including a comparator with hysteresis. The node will stop its operation when voltage falls below $1 \mathrm{~V}$, but it will not become active again until super-capacitor voltage exceeds a certain higher value (a threshold voltage).

To calculate this threshold voltage $\left(V_{\text {tresh }}\right)$, the capacity of the super capacitor must be taken into account, as well as the total amount of energy consumed during one cycle. Expression (8) calculates this value, and Figure 11 plots the voltage threshold for various super-capacitor capacities depending on the amount of energy consumed in one cycle.

$$
V_{\text {Thresh }}=V_{\text {off }}+\frac{1}{C} \sum_{i} I_{i} t_{i}
$$

where $C$ is the capacity of the super capacitor, $V_{\text {off }}$ is the minimum voltage at which the node can operate, and the sum represents the total aggregated energy demanded from the super capacitor during one cycle. This expression has been evaluated for different capacitor capacities and energy consumptions in Figure 11.

In Figure 12, the results of applying the hysteresis mechanism are shown for $m=5$, and it can be seen that the node is able to start operation and performs as expected. The hysteresis threshold in this experiment is set at $1.1 \mathrm{~V}$. Although a lower voltage threshold could be used according to expression (8) and Figure 11, 1.1 V was chosen to provide a security buffer.

Aside from studying the different energy-depletion scenarios and unrecoverable energy failures, simulations can be very useful to decide the order of magnitude of system components for a given application. For example, available energy is directly proportional to capacitance. In addition, prices are related to this capacitance value, and so making a realistic estimation of the system components is very important when planning the deployment of a WSN.

As an example, Figure 13 depicts a comparison between capacitors of 5 and 10 farads. Although our previous experiments were conducted with a 50-farad super capacitor, it can be observed that using a 10-farad capacitor may suffice. Moreover, different solar panels can be tested via simulation, thereby helping to better tune the hardware nodes to the application requirements. Carrying out this type of study when planning to deploy a WSN can led to important savings in hardware costs. 


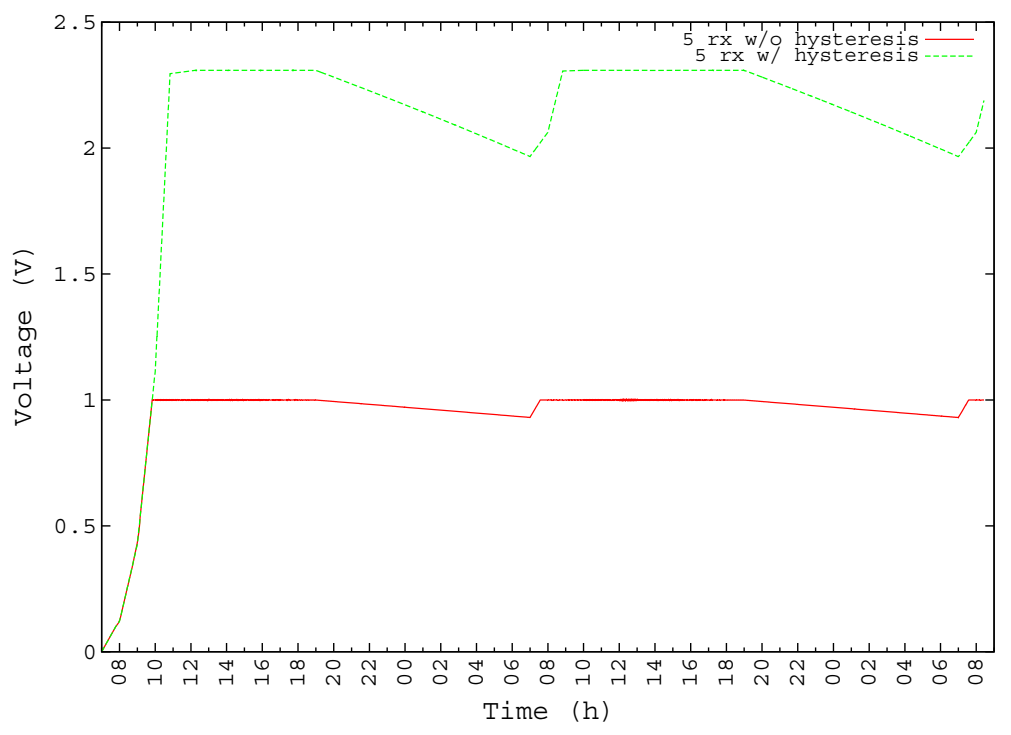

Figure 12. Increasing the RX time by $m=5$ times with and without hysteresis.

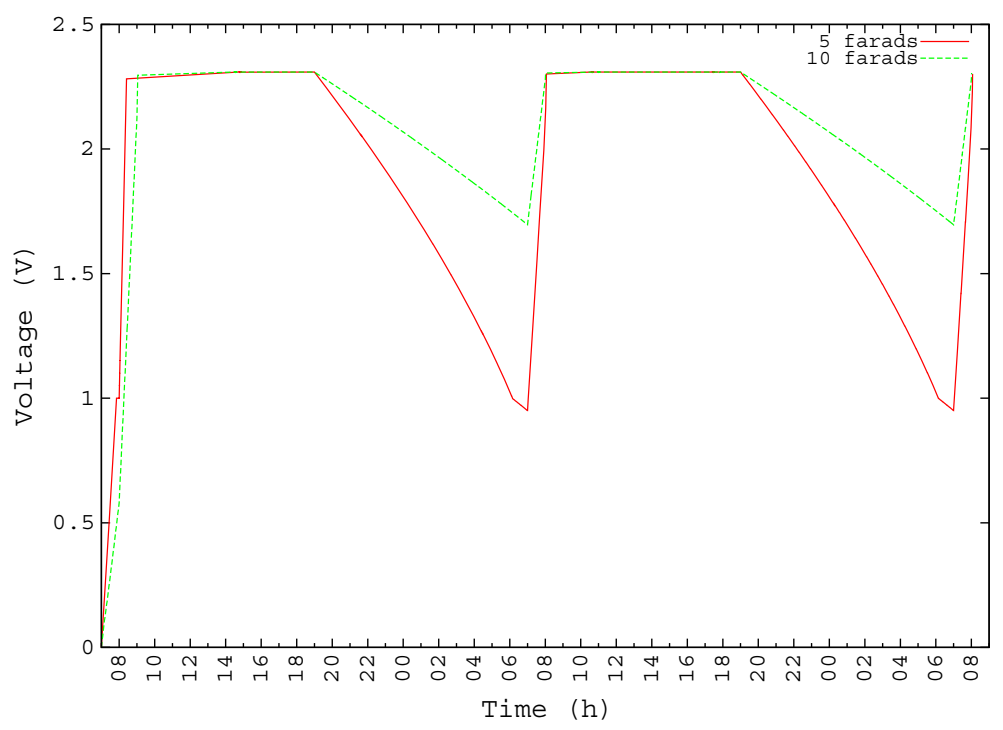

Figure 13. Capacitance variation.

\section{SIMPLE ENERGY-AWARE POLICIES}

Previously, it was shown how an unrecoverable energy node failure can be easily solved using a simple mechanism such as hysteresis. In this section, the proposed model is used to show how an energy-aware policy can help obtain more statistically meaningful data.

To accomplish this, a 1-year long period is going to be simulated in two different locations using solar radiation data from the PANGAEA [9] project. The chosen locations were Toravere in Estonia during 2011 and Barrow in Alaska (USA) during 2009. Their solar radiation values are depicted in Figure 14.

The study case is as follows. Nodes perform as specified in Figure 5; they will only carry out one transmission cycle $(n=0, m=0)$ and their sleep period is $5 \mathrm{~s}$. Power consumption parameters are the same as those specified in Section 5. 


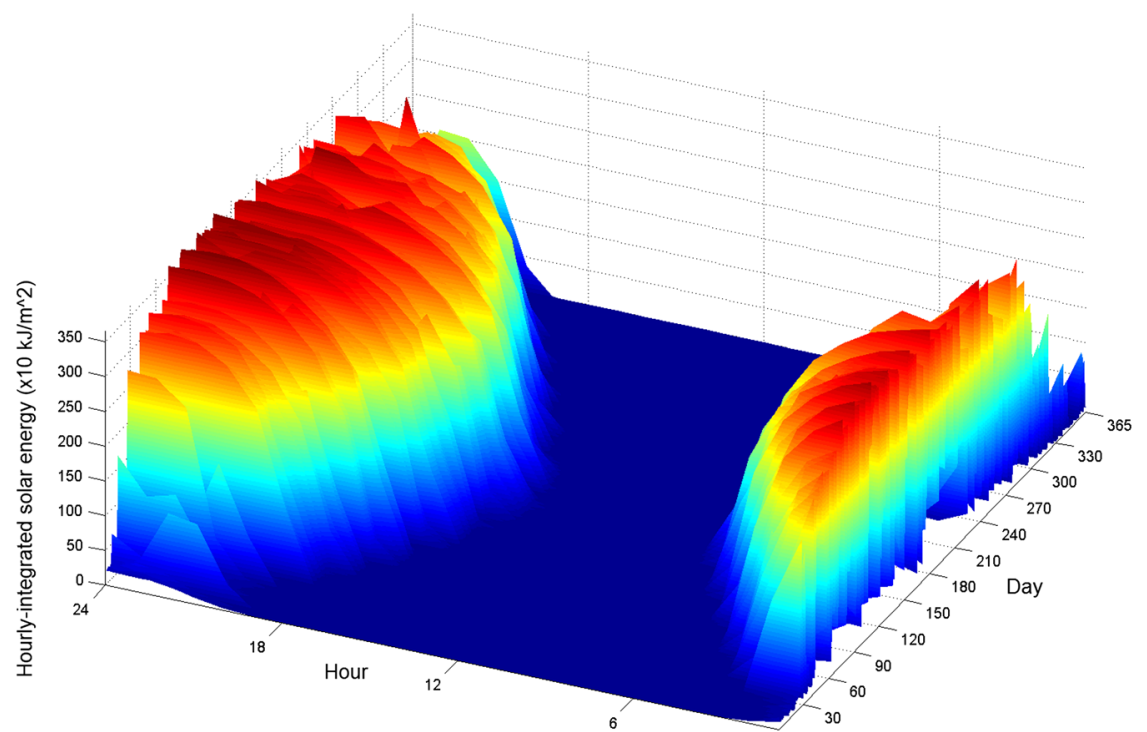

(a) Tõravere (EE) year 2011

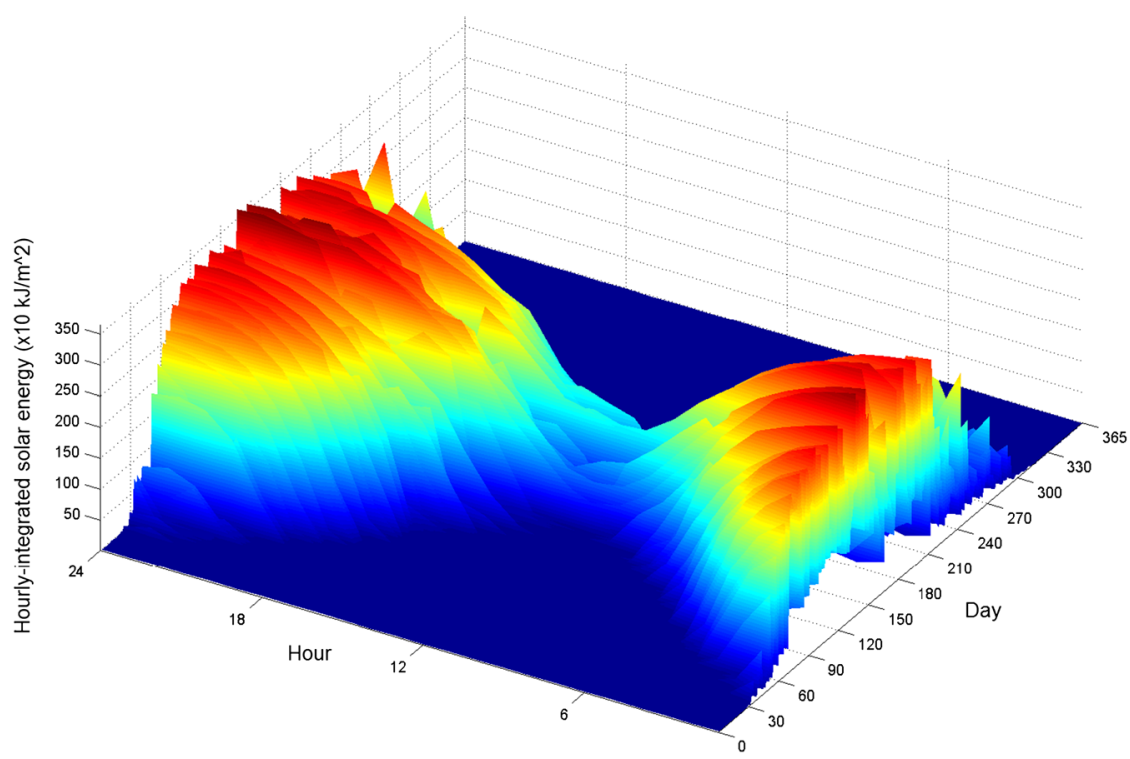

(b) Alaska (US) year 2009

Figure 14. Twenty-four hours, 365 days solar radiation integrated energy $\left(x 10 \frac{\mathrm{kJ}}{\mathrm{m}^{2}}\right)$. Zero hour corresponds to maximum solar power time. Day 0 corresponds to January 1. (a) Tõravere (EE) year 2011; (b) Alaska (USA) year 2009.

Figure 15 depicts the capacitor's maximum and minimum voltage for each day of the year, in the two specified locations. In the Alaska scenario, during the first and the last days of the year, there is not enough solar radiation to charge the capacitor, and so the node remains disabled. When the capacitor voltage is higher than $1.1 \mathrm{~V}$, the node starts its operation and is disconnected below $1 \mathrm{~V}$. This means that the days when the minimum voltage is lower than one, but the maximum voltage is higher, the node is disabled for a certain amount of time, usually during the night, without sending data to the sink. This behavior can lead to several hours passing without information being received, and so isolated islands in the network are created.

Table IV, in the 'Regular' columns, shows the packet statistics for the two locations compared with the optimum case (in which nodes always have enough energy to send their data). The first 


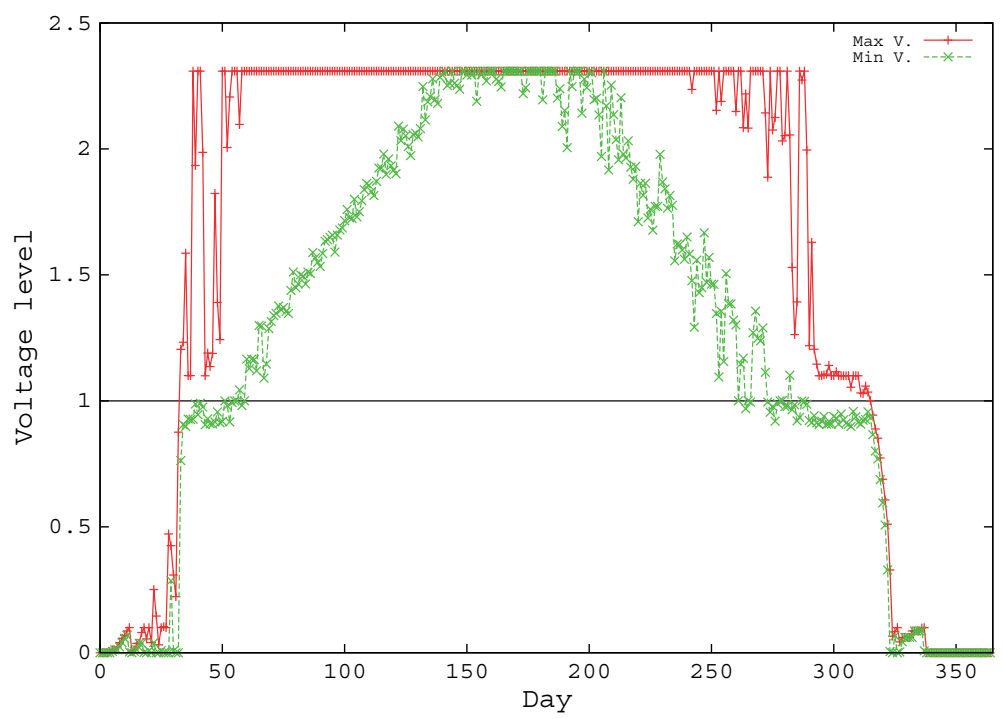

(a) Barrow (Alaska)

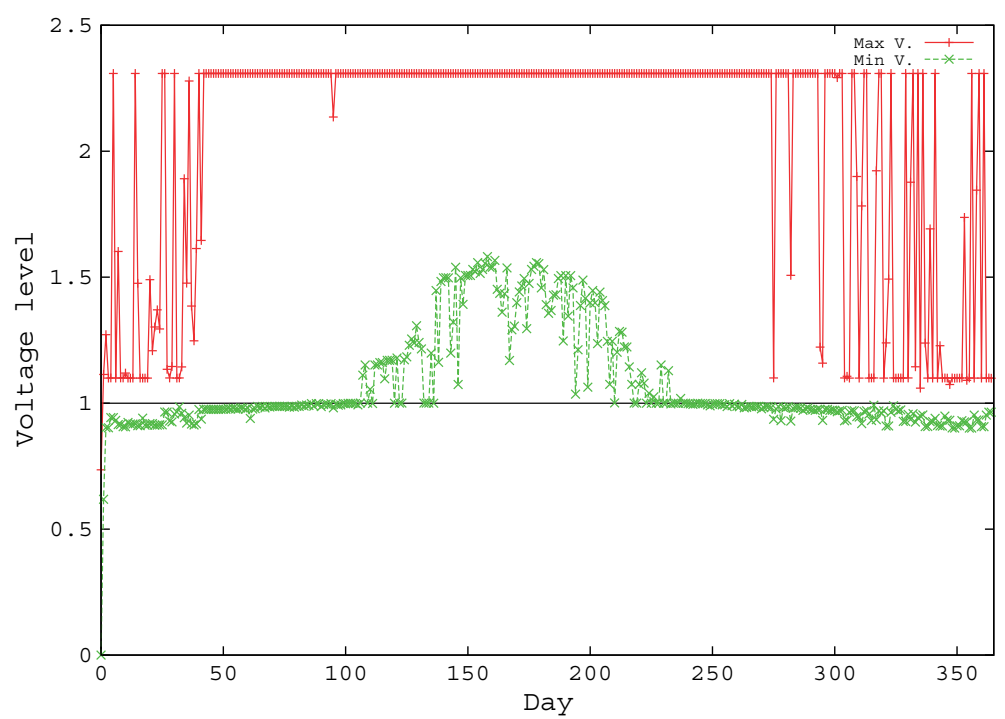

(b) Tõravere (Estonia)

Figure 15. Node max. and min. voltages during 1 year. (a) Barrow (Alaska); (b) Tõravere (Estonia).

Table IV. Packet statistics with and without energy-aware policy in two different locations.

\begin{tabular}{|c|c|c|c|c|c|c|c|c|c|}
\hline & \multirow[b]{3}{*}{ Optimum } & \multicolumn{4}{|c|}{ Alaska } & \multicolumn{4}{|c|}{ Estonia } \\
\hline & & \multicolumn{2}{|c|}{ Regular } & \multicolumn{2}{|c|}{ Policy } & \multicolumn{2}{|c|}{ Regular } & \multicolumn{2}{|c|}{ Policy } \\
\hline & & Value & Dev. (\%) & Value & Dev. (\%) & Value & Dev. $(\%)$ & Value & Dev. (\%) \\
\hline Total & $6,127,029$ & $4,051,986$ & 34 & $4,058,898$ & 34 & $5,067,768$ & 17 & $5,049,840$ & 18 \\
\hline IAT packets & 5.12 & 5.91 & 15 & 5.95 & 16 & 6.19 & 20 & 6.22 & 21 \\
\hline STDEV packets & 0 & 231.94 & - & 50.45 & 78 & 267.69 & - & 58.37 & 78 \\
\hline
\end{tabular}

IAT, interarrival time; STDEV, standard deviation.

thing to notice is that the number of transmissions and receptions in both scenarios is lower than the optimum because there are periods in which the node has not enough energy to operate. As can be seen in Estonia, the mean interarrival time (IAT) between transmissions and receptions is almost 
$20 \%$ higher than the optimum case - and the standard deviation (STDEV) of the mean IAT is also high. This implies that no data are received for long periods of time.

To solve this issue, there has been some research effort to design adaptive power management systems [32-34]. These approaches, although very efficient, are rather complex and might be difficult to implement in an 8-bit low-power resource-constrained microcontroller.

Instead, in equation (9), we propose a simple but effective energy-aware policy that modifies the predefined sleep time according to the voltage remaining in the capacitor. This equation only needs to store in memory the previous value of the capacitor voltage - which can be easily obtained using an Analog-to-Digital Converter (ADC) converter.

$$
S_{t}= \begin{cases}S_{\text {min }} & \text { if } V_{t} / V_{0}>\Theta \\ \left.\max S_{\text {min }}, \frac{S_{t-1}}{\arctan \left(\left(\frac{\Delta V_{t}}{V_{t-1}}+\frac{V_{t}}{V_{0}}\right) \times \alpha\right)} \times \beta\right) & \text { if } \Delta V \geqslant 0 \\ \min \left(S_{\text {max }}, S_{t-1} \times \arctan \left(\left(\frac{\left|\Delta V_{t}\right|}{V_{t}}+\frac{V_{t}}{V_{0}}\right) \times \alpha\right) \times \beta\right) & \text { if } \Delta V<0\end{cases}
$$

In this equation, $S_{\min }$ and $S_{\max }$ are the minimum and maximum sleep time periods given by the requirements of the application. $S$ is the current sleep time. $V_{0}$ is the maximum current delivered by the capacitor, and $V_{t}$ and $V_{t-1}$ are the current and previous voltages, respectively. The constant $\Theta$ defines the voltage threshold value on which the policy will be executed. Finally, $\alpha$ and $\beta$ are two configurable constants that enable an adjustment of the function sensitivity to the application requirements.

By modifying the time a node remains in sleep state (i.e., the duty cycle), this strategy distributes the energy more efficiently and avoids long periods without data collection. Let us suppose a super-capacitor energy level and the impossibility of collecting harvested energy (at night for example). Using a good policy, the total number of transmitted packets during this time should be approximately equal to the case when no policy is applied. However, the STDEV of the IAT must considerably decrease.

The effect of this policy on the simulations can be seen in Figure 16, where the parameters of the function were found experimentally and are shown in Table V. In the scenario in Alaska, Figure 16(a), the policy has no effect on the first and last days of the year because there is not enough energy. But when the node starts working, the policy is able to maintain the node in the working region continuously during more than 300 days compared with 200 days in the scenario without policy.

The same occurs at the scenario in Estonia, Figure 16(b). When comparing the voltage values for the scenario without the proposed policy, Figure 15(b), and the policy that implements the policy, Figure 16(b), it can be seen how, by using the policy, the node is able to stay in the working region during the entire year.

Table IV includes the packet statistics for the scenarios using the proposed policy. It can be seen that the policy extends the node working time and distributes the transmissions more efficiently. As stated before, the total number of transmissions should be approximately the same with or without the policy, so mean IAT stays constant in any case. The objective of the presented policy is to communicate with the node with certain predictability and avoid long time gaps, which is accomplished by deceasing STDEV values. In both cases, this value is reduced by around $78 \%$, and so it can be expected that node activity gaps are reduced to one fourth - with one transmission cycle almost every minute.

The effect of the policy can also be seen in Figure 17, where the distribution of periods without messages sent is plotted according to the voltage level of the super capacitor. Figure 17(a) shows this distribution without using any policy. As can be seen, until the threshold voltage at which the node stops functioning is reached, packets are sent every $5 \mathrm{~s}$. When this threshold voltage is reached, the node stops its operation and waits between 1 and $60 \mathrm{~h}$, until there is enough power to restart transmission.

In Figure 17(b), this distribution is depicted when the proposed policy is applied. It can be seen that the maximum interval without sending messages is $1 \mathrm{~h}$, which is the configured maximum interval for the policy. Moreover, the configured threshold at which the policy starts to be applied can be 


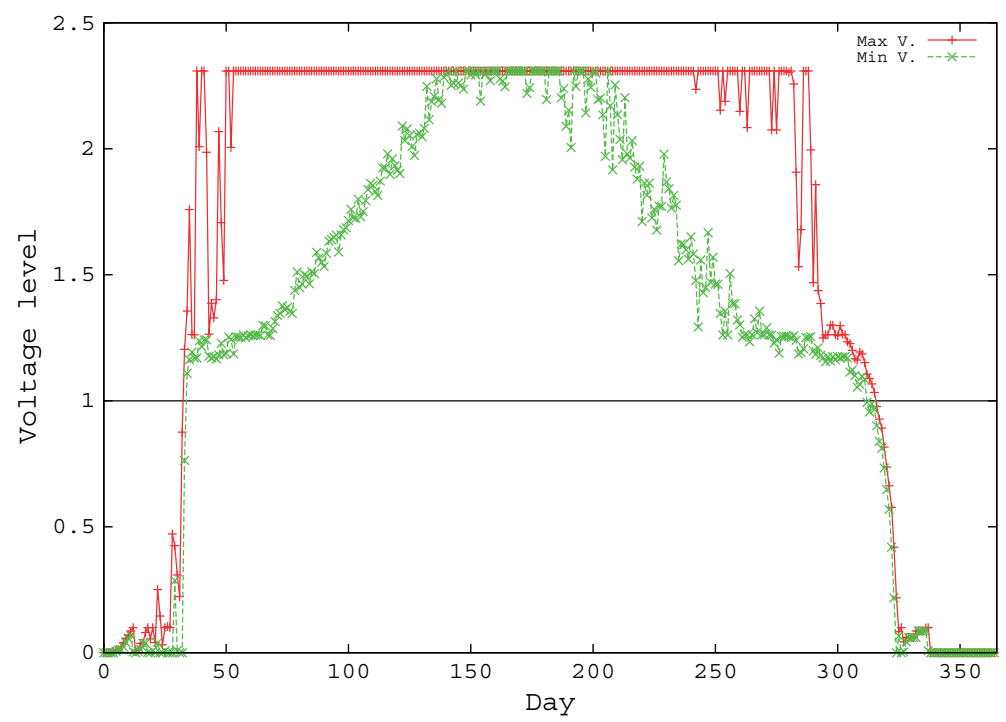

(a) Barrow (Alaska)

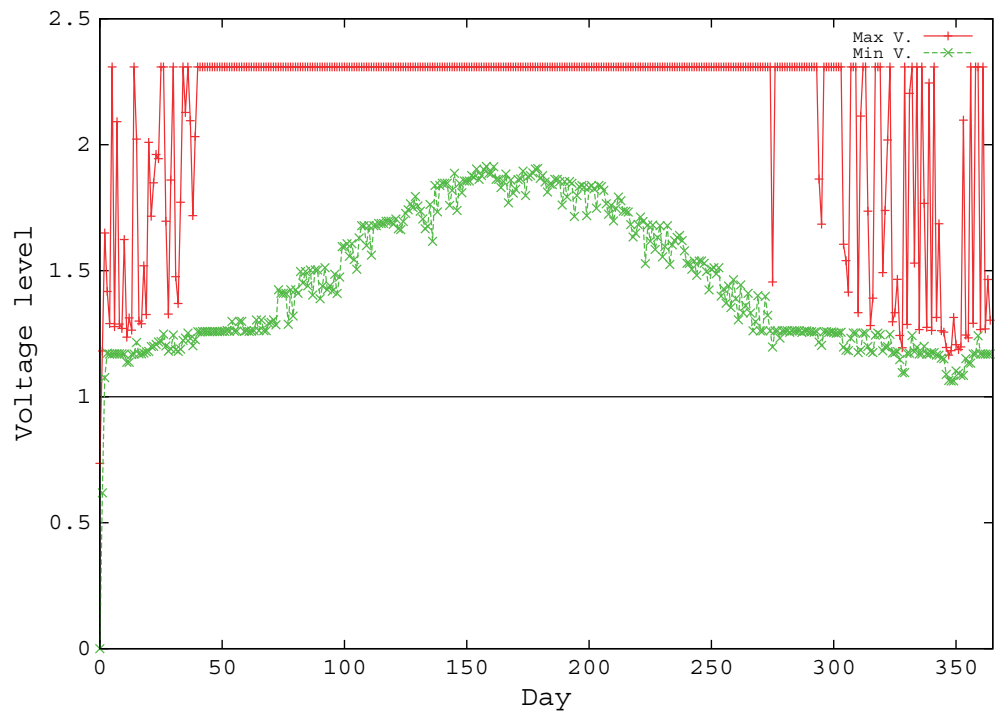

(b) Tõravere (Estonia)

Figure 16. Node max. and min. voltages when applying the proposed policy during 1 year. (a) Barrow (Alaska); (b) Tõravere (Estonia).

Table V. Policy variables values.

\begin{tabular}{lc}
\hline Variable & Value \\
\hline$S_{\min }$ & $5 \mathrm{~s}$ \\
$S_{\max }$ & $3600 \mathrm{~s}$ \\
$V_{0}$ & $2.3 \mathrm{~V}$ \\
$\Theta$ & 0.2 \\
$\alpha$ & 10 \\
$\beta$ & 2 \\
\hline
\end{tabular}

clearly seen, and it is also possible to see how it is able to distribute the periods without messages in order to reduce the STDEV of the IAT.

In the Alaska case - with no solar radiation during several days - a backup battery is mandatory to guarantee node operation during the whole year. Even when super-capacitor voltage is below $1 \mathrm{~V}$ 


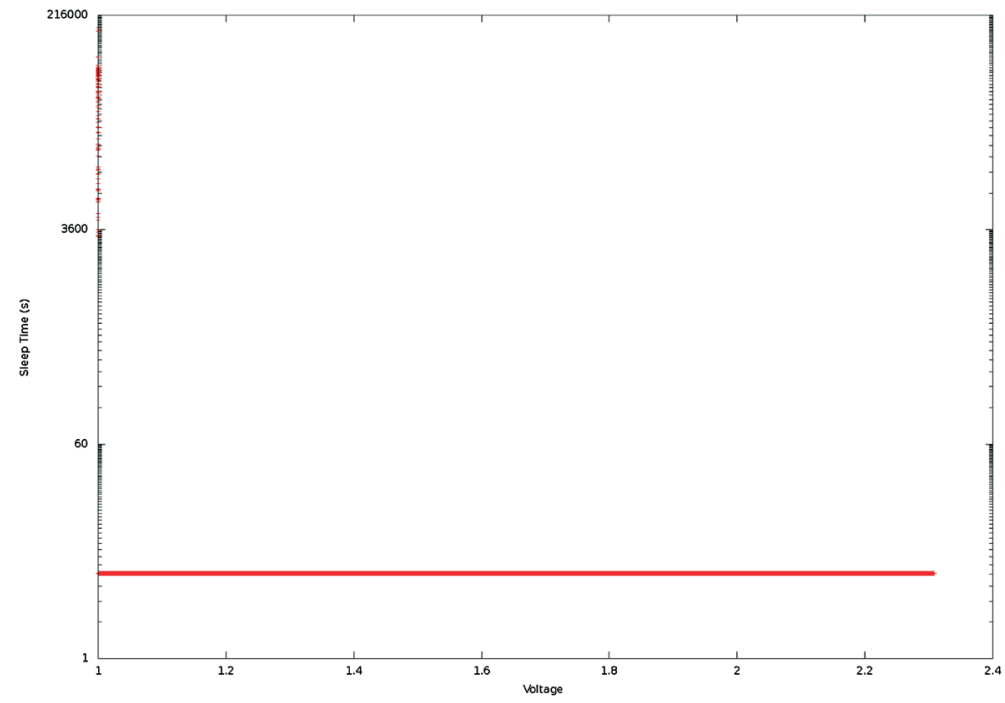

(a) Normal

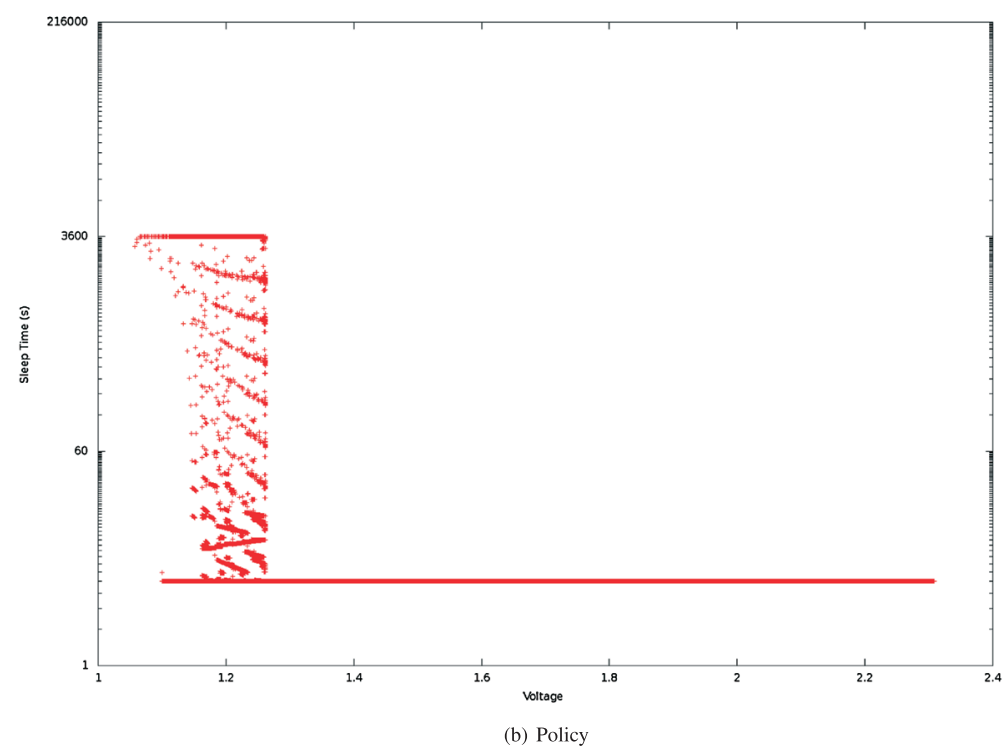

Figure 17. Voltage level and sleep time. (a) Normal; (b) policy.

(node is completely shut down, and overall consumption is a few $\mu \mathrm{A}$ ), its energy still decreases until it is depleted. No policy can be applied to extend node life only using super capacitors. In future work, automatic battery-backup switching will be modeled and evaluated, because the hardware platform implements this feature.

\section{CONCLUSIONS}

In this paper, a new energy model for the ns-3 simulator using super-capacitor energy storage with solar energy-harvesting recharge has been presented. The model is dynamic because it considers radio mode changes and solar radiation variations per hour. This innovation is very important for future network designs because it makes it possible to simulate the impact of radiation variability during several days, weeks, or months. Moreover, solar radiation values can be easily imported from public meteorological databases in different world locations. As an example, this work uses the records of the AEMet Spanish office and the PANGAEA international initiative. 
The presented experiments are focused on validating the solar panel energy model, the capacitor energy model, and the node consumption model. Results show a good correlation between the simulations and real experiments.

In addition, it has been shown how this model can provide assistance during the design phases by testing different component magnitudes before deploying the network. Therefore, it opens the possibility of reducing complexity and prices at very early stages of the network design by taking into account the future geographical location.

The preliminary experiments conducted reveal the need for intelligent energy control during critical solar periods. In a first approximation, a comparator with hysteresis is introduced - which prevents the operational death of the node. Eventually, an adaptive energy-aware policy has been introduced that enables the operational period of the node to be extended during long and critical solar periods by adjusting the duty cycle. In an environmental data monitoring application, this can help improve the collected data by avoiding a node total failure and so retrieve more statistically meaningful data.

Generally speaking, this research can assist in the study and development of energy-efficient algorithms that offer energy-neutral operation and dynamic energy adjustment in order to build everlasting WSNs.

As future work, we plan to further extend the model to include a backup energy store such as a battery. Moreover, we intend to add new energy-harvesting sources such as wind or vibration and test the performance of various energy-aware routing and medium access protocols for WSN.

\section{ACKNOWLEDGEMENTS}

This work was supported by the CICYT (research projects CTM2011-29691-C02-01 and TIN2011-28435C03-01) and UPV research project SP20120889.

\section{REFERENCES}

1. Akyildiz IF, Vuran Can M. Wireless Sensor Networks. John Wiley \& Sons: New York City, 2010.

2. Seah W., Tan YK, Chan A. Research in energy harvesting wireless sensor networks and the challenges ahead. In Autonomous Sensor Networks SE - 27, Vol. 13, Filippini D (ed.), Springer Series on Chemical Sensors and Biosensors. Springer Berlin Heidelberg: Berlin, 2013; 73-93.

3. Vullers R, Schaijk R, Visser H, Penders J, Hoof C. Energy harvesting for autonomous wireless sensor networks. IEEE Solid-State Circuits Magazine 2010; 2(2):29-38.

4. Ammar Y, Buhrig A, Marzencki M, Charlot B, Basrour S, Matou K, Renaudin M. Wireless sensor network node with asynchronous architecture and vibration harvesting micro power generator. In Proceedings of the 2005 Joint Conference on Smart Objects and Ambient Intelligence Innovative Context-Aware Services: Usages and Technologies sOc-EUSAI '05. ACM Press: New York, New York, USA, 2005; 287.

5. Vijayaraghavan K, Rajamani R. Active control based energy harvesting for battery-less wireless traffic sensors. In 2007 American Control Conference. IEEE: New York, 2007; 3106-3111.

6. Bottner H, Nurnus J, Gavrikov A, Kuhner G, Jagle M, Kunzel C, Eberhard D, Plescher G, Schubert A, Schlereth K. New thermoelectric components using microsystem technologies. Journal of Microelectromechanical Systems June 2004; 13(3):414-420.

7. Mateu L, Codrea C, Lucas N, Pollak M, Spies P. Energy harvesting for wireless communication systems using thermogenerators. Conference on Design of Circuits and Integrated Systems (DCIS), Barcelona, Spain, 2006.

8. AEMet. Agencia Estatal de Meteorolgía, 2013. Available from:http//www.aemet.es.

9. PANGAEA. Data Publisher for Earth \& Environmental Science, 2013. Available from:http://www.pangaea.de/.

10. Merrett G, Weddell A, Lewis A, Harris N, Al-Hashimi B, White N. An empirical energy model for supercapacitor powered wireless sensor nodes. In 2008 Proceedings of 17th International Conference on Computer Communications and Networks. IEEE: St. Thomas U.S. Virgin Islands, 2008; 1-6.

11. Raghunathan V, Kansal A, Hsu J, Friedman J, Srivastava M. Design considerations for solar energy harvesting wireless embedded systems. In IPSN '05 Proceedings of the 4th International Symposium on Information Processing in Sensor Networks. IEEE Press: Los Angeles, California, 2005; 64.

12. Zeng K, Ren K, Lou W, Moran PJ. Energy aware efficient geographic routing in lossy wireless sensor networks with environmental energy supply. Wireless Networks February 2007; 15(1):39-51.

13. Hasenfratz D, Meier A, Moser C, Chen JJ, Thiele L. Analysis, comparison, and optimization of routing protocols for energy harvesting wireless sensor networks. In 2010 IEEE International Conference on Sensor Networks, Ubiquitous, and Trustworthy Computing. IEEE: Newport Beach, California, 2010; 19-26. 
14. Noh DK, Hur J. Using a dynamic backbone for efficient data delivery in solar-powered WSNs. Journal of Network and Computer Applications July 2012; 35(4):1277-1284.

15. Lin L, Shroff NB, Srikant R. Asymptotically optimal energy-aware routing for multihop wireless networks with renewable energy sources. IEEE/ACM Transactions on Networking October 2007; 15(5):1021-1034.

16. Ferry N, Ducloyer S, Julien N, Jutel D. Power/energy estimator for designing WSN nodes with ambient energy harvesting feature. EURASIP Journal of Embedded Systems 2011; 2011:6:1-6:17.

17. Glaser J, Weber D, Madani S, Mahlknecht S. Power aware simulation framework for wireless sensor networks and nodes. EURASIP Journal on Embedded Systems June 2008; 2008(1):369-178.

18. Merrett GV, White NM, Harris NR, Al-Hashimi BM. Energy-aware simulation for wireless sensor networks. In 2009 6th Annual IEEE Communications Society Conference on Sensor, Mesh and Ad Hoc Communications and Networks. IEEE: Rome, 2009; 64-71.

19. De Mil P, Jooris B, Tytgat L, Catteeuw R, Moerman I, Demeester P, Kamerman A. Design and implementation of a generic energy-harvesting framework applied to the evaluation of a large-scale electronic shelf-labeling wireless sensor network. EURASIP Journal on Wireless Communications and Networking February 2010; 2010(1):343-690.

20. Castagnetti A. A framework for modeling and simulating energy harvesting WSN nodes with efficient power management policies. EURASIP Journal of Embedded Systems 2012; 2012(1):1-20.

21. Alippi C, Galperti C. An adaptive system for optimal solar energy harvesting in wireless sensor network nodes. Circuits and Systems I: Regular Papers, IEEE Transactions on 2008; 55(6):1742-1750.

22. Jiang X, Polastre J, Culler D. Perpetual environmentally powered sensor networks. In Information Processing in Sensor Networks, 2005. IPSN 2005. Fourth International Symposium on. IEEE: Los Angeles, California, 2005; 463-468.

23. Park C, Chou PH. Ambimax: autonomous energy harvesting platform for multi-supply wireless sensor nodes. In Sensor and Ad Hoc Communications and Networks, 2006. SECON'06. 20063 rd Annual IEEE Communications Society on, Vol. 1. IEEE: Reston, VA, 2006; 168-177.

24. Simjee F, Chou PH. Everlast: long-life, supercapacitor-operated wireless sensor node. In Low Power Electronics and Design, 2006. ISLPED'06. Proceedings of the 2006 International Symposium on. IEEE: Tegernsee, Germany, 2006; 197-202.

25. Sanchez A, Climent S, Blanc S, Capella JV, Piqueras I. WSN with energy-harvesting: modeling and simulation based on a practical architecture using real radiation levels. In Proceedings of the 6th ACM Workshop on Performance Monitoring and Measurement of Heterogeneous Wireless and Wired Networks - PM2HW2N'11. ACM Press: New York, New York, USA, 2011; 17-24.

26. Kansal A, Hsu J, Zahedi S, Srivastava MB. Power management in energy harvesting sensor networks. ACM Transactions on Embedded Computing Systems September 2007; 6(32).

27. Zhu T, Zhong Z, Gu Y, He T, Zhang ZL. Leakage-aware energy synchronization for wireless sensor networks. In Proceedings of the 7th International Conference on Mobile Systems, Applications, and Services, MobiSys '09. ACM: New York, NY, USA, 2009; 319-332.

28. Renner C, Jessen J, Turau V. Lifetime prediction for supercapacitor-powered wireless sensor nodes. Proc. of the 8th GI/ITG KuVS Fachgesprächi Drahtlose Sensornetze(FGSN09), Hamburg, Germany, 2009.

29. TI. Analog, Embedded Processing, Semiconductor Company, Texas Instruments, 2013. Available from:http//www. ti.com.

30. WSNVAL. Wireless Sensor Networks Valencia, 2013. Available from:www.wsnval.com.

31. Sanchez A, Blanc S, Yuste P, Serrano J. RFID based acoustic wake-up system for underwater sensor networks. In 2011 IEEE Eighth International Conference on Mobile Ad-Hoc and Sensor Systems. IEEE: Valencia, Spain, 2011; 873-878.

32. Fan KW, Zheng Z, Sinha P. Steady and fair rate allocation for rechargeable sensors in perpetual sensor networks. In Proceedings of the 6th ACM Conference on Embedded Network Sensor Systems - SenSys '08. ACM Press: Raleigh, NC, USA, 2008; 239-252.

33. Moser C, Thiele L, Brunelli D, Benini L. Adaptive power management for environmentally powered systems. IEEE Transactions on Computers April 2010; 59(4):478-491.

34. Zhang B, Simon R, Aydin H. Maximum utility rate allocation for energy harvesting wireless sensor networks. In Proceedings of the 14th ACM International Conference on Modeling, Analysis and Simulation of Wireless and Mobile Systems - MSWIM '11. ACM Press: Miami, Florida, USA, 2011; 7-16. 